\title{
The ATLAS Tile Calorimeter DCS for Run 2
}

\author{
F. Martins
}

on behalf of the ATLAS Tile Calorimeter System

IEEE NSS/MIC, Strasbourg - 29 October - 5 November, 2016 


\section{Overview}

- LHC and the ATLAS Detector

- TileCal hardware supervised by DCS

- TileCal DCS for Run 2

- Summary 


\section{Tile Calorimeter Detector (TileCal)}

- Designed for jet energy and ETmiss measurements

- $12 \mathrm{~m}$ length and outer radius of $4.25 \mathrm{~m}$

- Weights 2900 tons

- Uses steel plates as passive absorber and scintillating tiles as active material read by WLS optical fibers
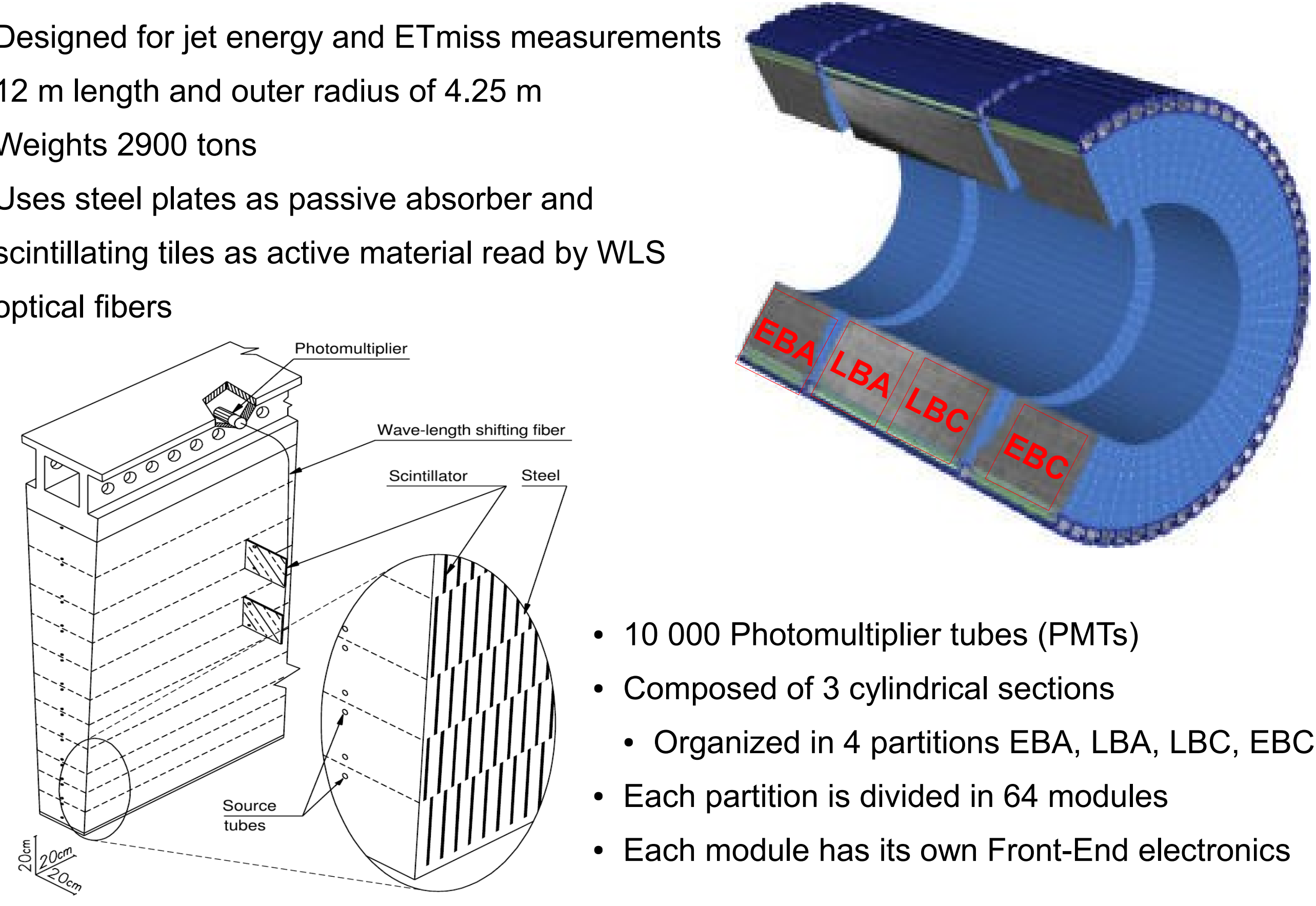

- 10000 Photomultiplier tubes (PMTs)

- Composed of 3 cylindrical sections

- Organized in 4 partitions EBA, LBA, LBC, EBC

- Each partition is divided in 64 modules

- Each module has its own Front-End electronics 


\section{TileCal hardware supervised by DCS}

- Front-End (FE)

- Finger Low Voltage Power Supplies (FLVPS)

- Front End High Voltage distribution system

- High Voltage distribution cards (HVopto)

- High Voltage control cards (HV Micro)

- Back End (BE)

- Auxiliary Boards (AUXBoards)

- High Voltage Power supply

- 200V DC Power Supply

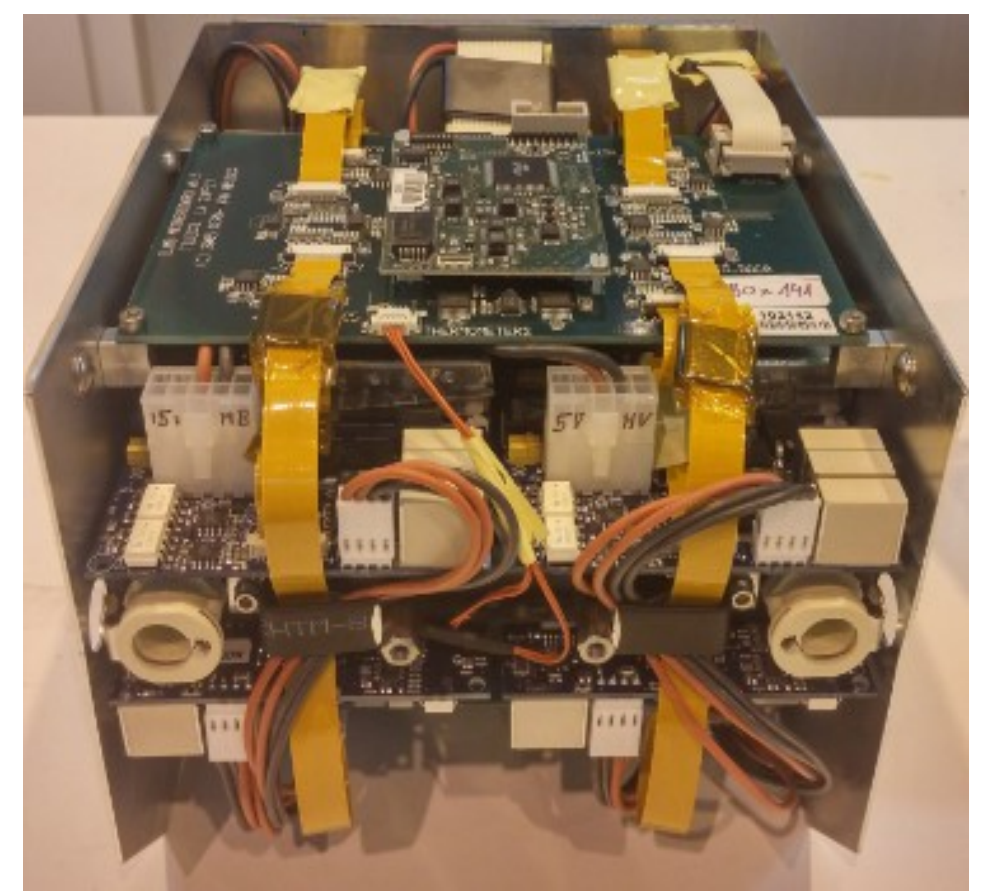

Finger Low Voltage Power Supply

- Low Voltage crates (Wiener) for VME Timing, Trigger and Control (TTC) and Read Out Driver boards (ROD)

- CANbus power supplies (CAN PS)

- Cooling system

- Laser system 


\section{TileCal Detector Control System}

The TileCal DCS "ensures the coherent and safe operation" of the detector

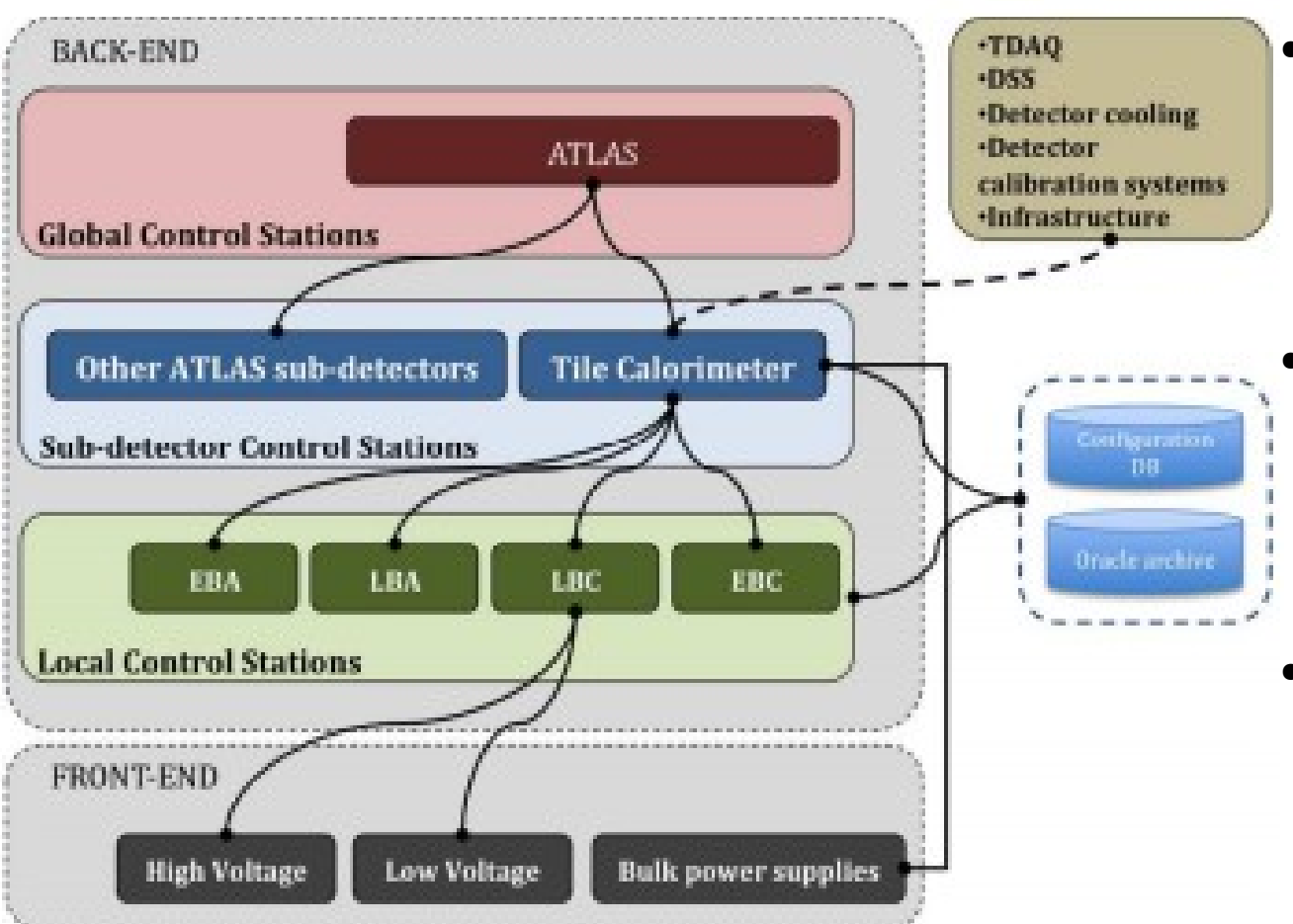

- Based on Supervision Control and Data Acquisition (SCADA) system - PVSS (Datapoints, managers, alarm, archiving, etc.)

- Joint Control Project (JCOP) framework and ATLAS central DCS provides common software tools and control for all detectors

- The data is exchanged via Distribution Information Management (DIM), OLE for Process Control (OPC) and Modbus

- Finite State Machine (FSM)

\begin{tabular}{l|l|c|c|}
\hline \multirow{2}{*}{ TIL } & READY & OK & $\unlhd$ \\
\hline EBA & READY & OK & $\unlhd$ \\
\cline { 2 - 4 } LBA & READY & OK & $\ominus$ \\
\cline { 2 - 4 } LBC & READY & OK & $\ominus$ \\
\cline { 2 - 4 } $\begin{array}{l}\text { EBC } \\
\text { INFRASTRUCTURE }\end{array}$ & READY & OK & $\unlhd$ \\
\cline { 2 - 4 } & READY & OK & $\unlhd$ \\
\hline
\end{tabular}

- Combines all the BE functional layers

- Allows full operation of the detector (control and monitoring) 


\section{TileCal DCS for Run 2}

- Follows the ATLAS DCS guidelines for software and hardware for Run 2

- Migration from Windows to CERN Scientific Linux 6

- Update WinCC Open Architecture (former PVSS)

- Migration from OPC Data Access (DA) and other middleware to OPC Unified Architecture (UA)

- Control system components of infrastructure located in old "TileCal" Sub-detector Control Station (SCS) were moved to a dedicated

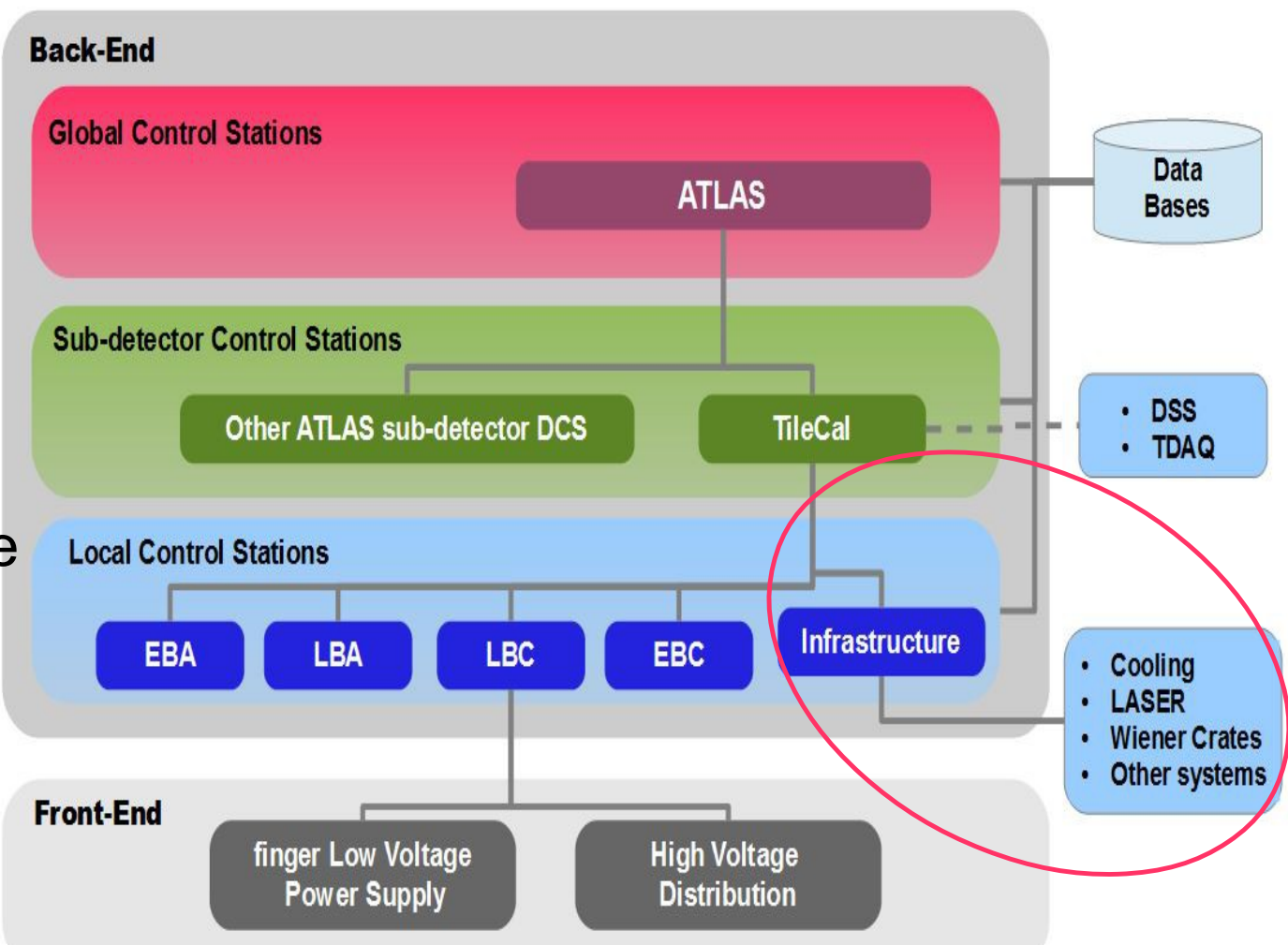
project - "Infrastructure"

- Detector requirements drove the developments of controls for the new hardware 


\section{Update of $200 \mathrm{~V}$ system}

Used to provide the 200V DC voltage to the Front-End power supplies.

- Replacement of 200V hardware as preventive action against hardware failures

TDK Lambda as new 200V DC

- One channel per unit capable of delivering $2.4 \mathrm{~kW}$

- Kept same granularity (1 unit per 4 FE )

- Development of control system

- Configured in 4 daisy-chains with 16 units each

- Stable Monitoring rate is $6 \mathrm{~s}, 3 \mathrm{x}$ times faster then before

- New user interfaces

- Merged with existing 200V control system

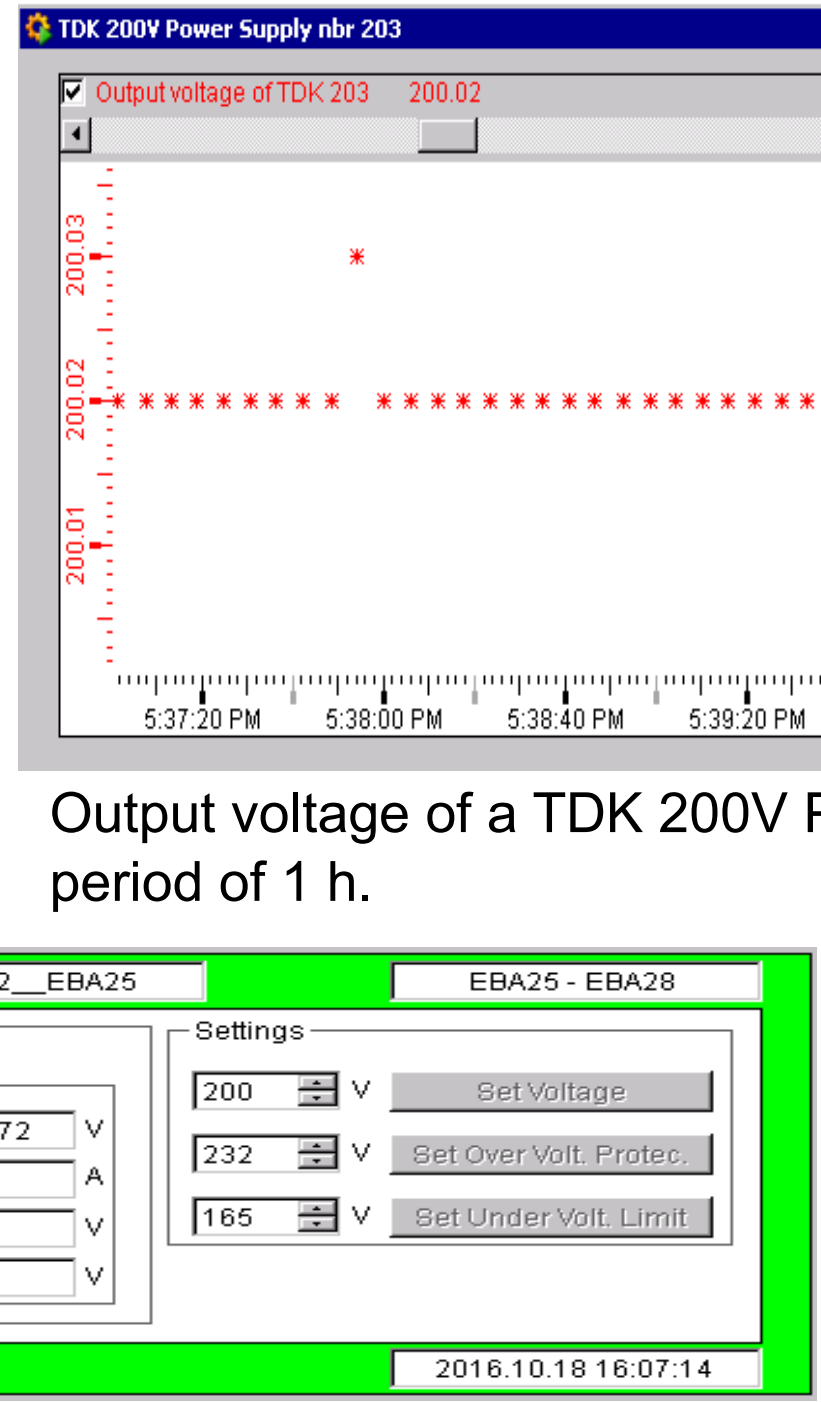

Example of user interface for new 200V PS 


\section{Upgrade of the OPC Data Access (DA) to Unified Architecture (UA)}

OPC UA is slimmer, multiplatform, allows securing traffic and source code is available!

It is used mainly for communication with:

- Embedded Local Monitoring Board (ELMB) - general purpose Input/Output board used in:

- FLVPS

- LV CANbus power supplies

- Auxiliary Boards

- Wiener VME Crates

Respective control systems were updated

- OPC server (provided by ATLAS central Team)

- Control procedures, scripts and libraries

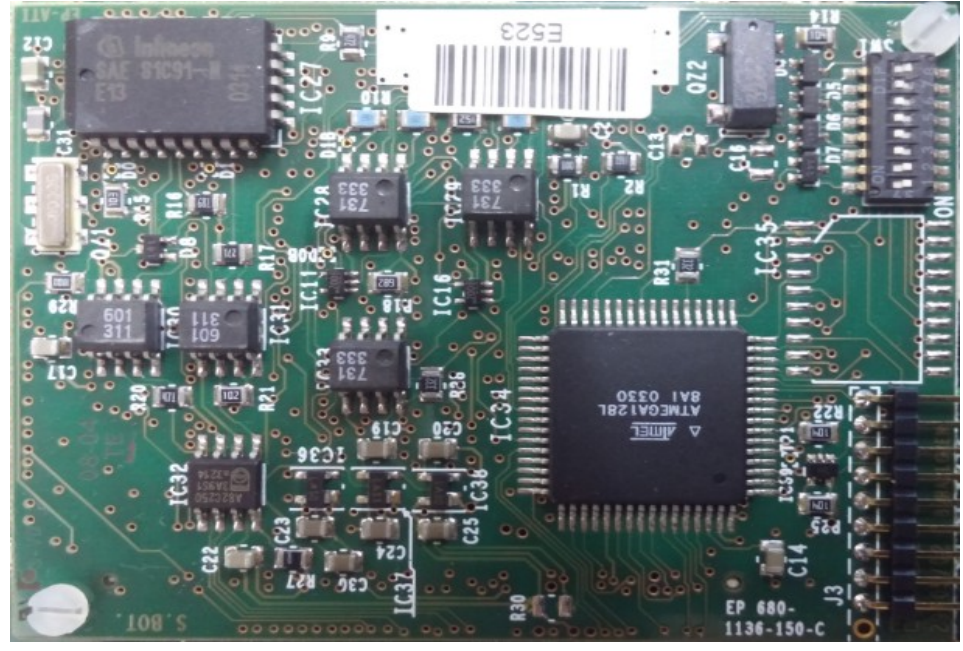

ELMB Board

Additionally in VME crates control system

- More crates were added

- Voltage and current monitoring for each crate channel 


\section{Control System Upgrade for HV-Micro}

- HV Micro are controller boards for:

- Regulation and monitoring of HV to the PMTs

- Monitoring of HV CANbus power supplies

- CANbus communication using custom made protocols (different for each type of device)

- HV Micro control system migrated from DIM-based

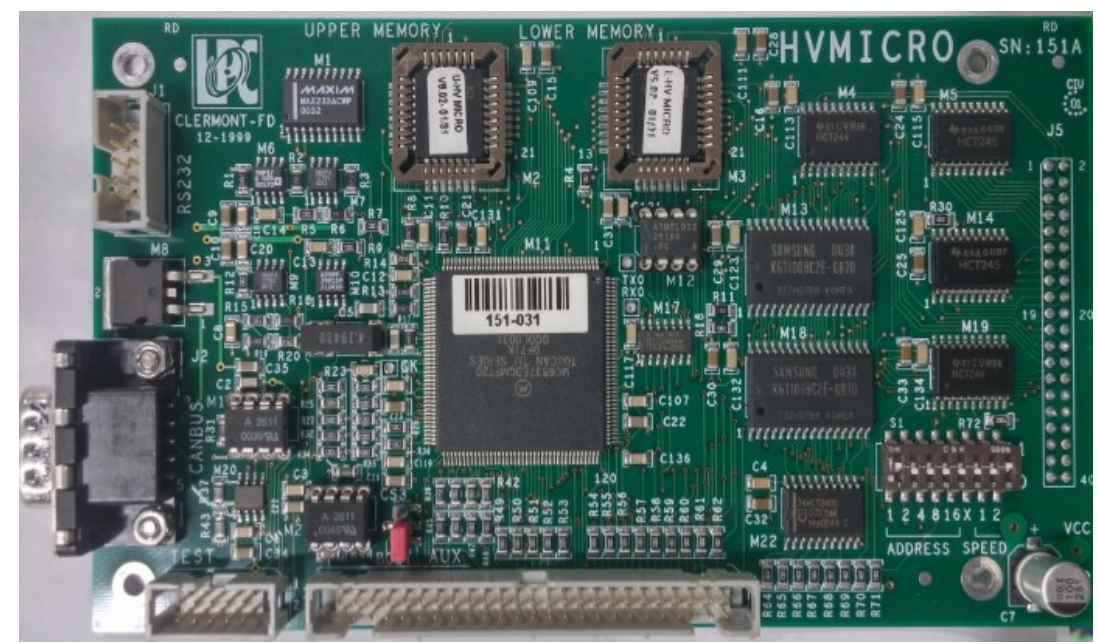

HV Micro board server to OPC UA

- Advantages of the OPC UA server relatively to DIM-based servers:

- Easier to maintain

- Usage of flags for invalid data (ex. reply timeout) made the system more reliable

- Includes additional control for the HV CAN PS

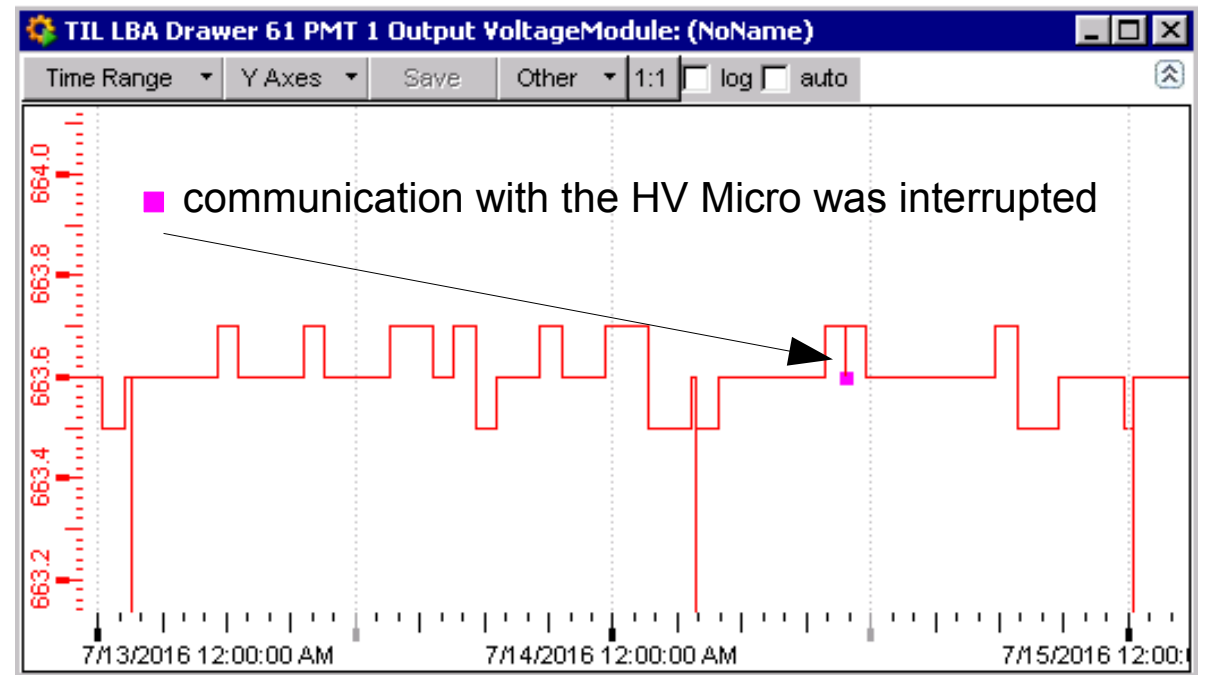

High Voltage applied to one PMT 


\section{Laser II system}

\section{TileCal make use of a laser system to monitor the stability of the PMTs signal}

The goal of the Laser upgrade is to reach the sub-percent level in the monitor of PMT signal

Stability of PMT response affected by:

- Radiation effect

- Temperature

- Drift of applied High Voltage

Development of new DCS consisted of:

- DIM server using a custom made protocol over TCP/IP

- New datapoint structure in WinCC

- Finite State Machine

- User interfaces

- Alarms

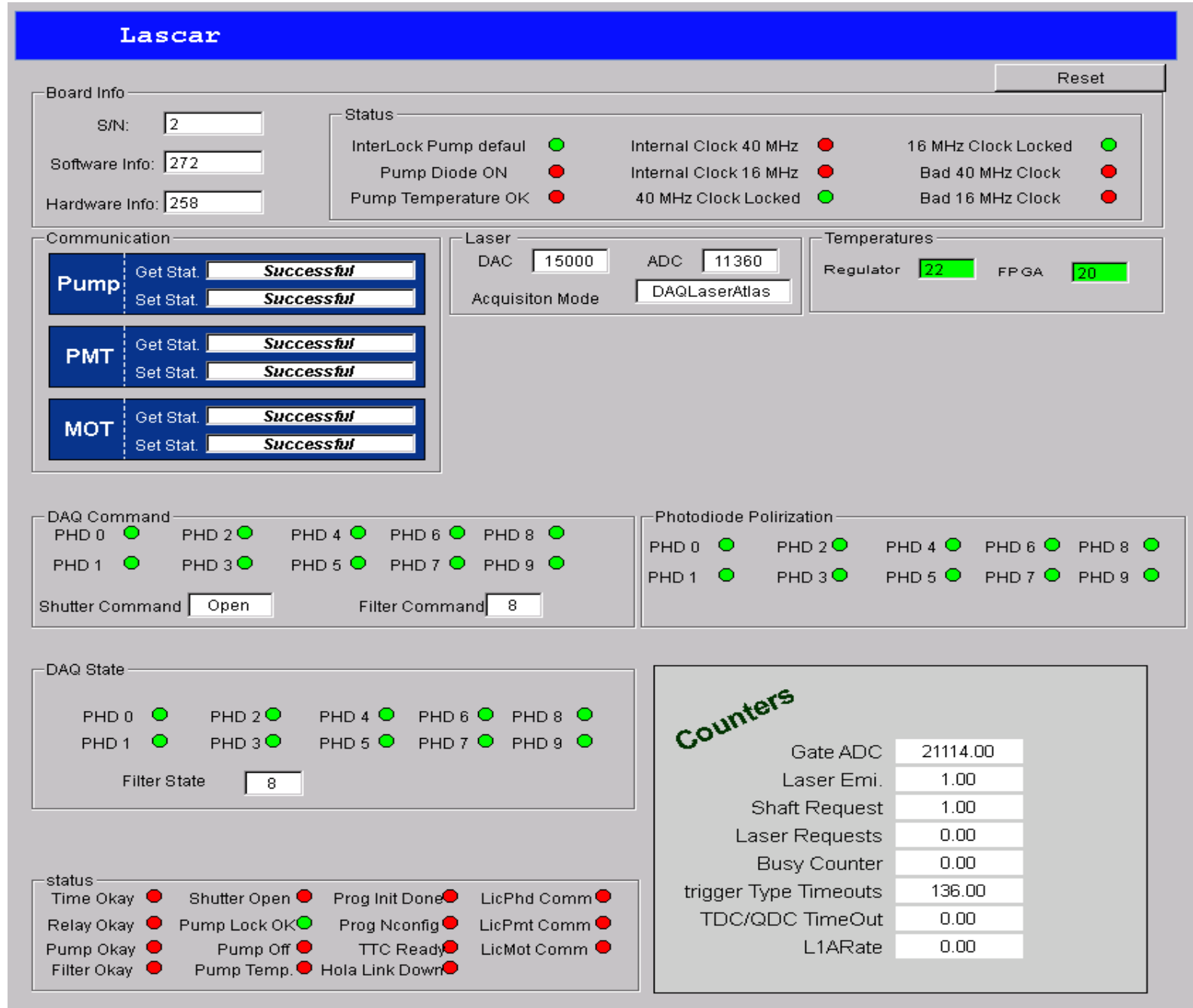

\section{Example of a new Laser II user interface}




\section{Summary}

- Migration of DCS control system from Windows to CERN Scientific Linux 6 allows an easier maintenance

- Development of OPC UA server allows to implement new functionalities, such as usage of the same server for communication with the HV Micro cards and of invalid bit for monitored data, making the system more reliable

- Some of the components from old "mixed" Sub-detector Control Stations were moved to a dedicated Infrastructure project which led to an uniformity of the DCS structure

- Development and implementation of DCS for Laser II system and 200V DC system (3x times faster) was done using new structure, excluding the OPC

The upgraded DCS is being used in Run 2 with sucess! 


\section{Acknowledgments}

Over the years several persons contributed to the development and maintenance of TileCal DCS. The actual status of the TileCal DCS would not be achieved without their work. To all of them a huge THANK YOU!

I would also like to thank ATLAS central DCS team for their contributions and support. A big thank to the colleagues of LIP and TileCal for their help and suggestions.

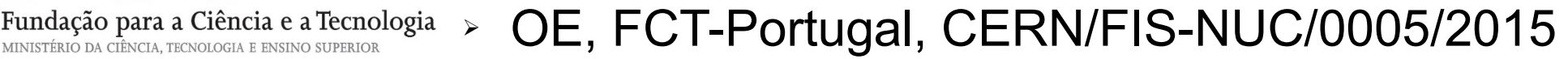

